\title{
Sea cucumber (Stichopus hermanii) based hydrogel to treat burn wounds in rats
}

\begin{abstract}
Malaysian sea cucumber was incorporated into hydrogel formulation by using electron beam irradiation technique and was introduced as novel cross-linked Gamat Hydrogel dressing. This study investigated whether Gamat Hydrogel enhanced repair of deep partial skin thickness burn wound in rats and its possible mechanism. Wounds were treated with either Gamat Hydrogel, control hydrogel, OpSite ${ }^{\circledR}$ film dressing or left untreated. Skin samples were taken at 7, 14, 21, and 28 days post burn for histological and molecular evaluations. Gamat Hydrogel markedly enhanced wound contraction and improved histological reorganization of the regenerating tissue. Furthermore, the dressing modulated the inflammatory responses, stimulated the activation and proliferation of fibroblasts, and enhanced rapid production of collagen fiber network with a consequently shorter healing time. The level of proinflammatory cytokines; IL-1Ŭ, IL-16, and IL-6, were significantly reduced in Gamat Hydrogel treated wounds compared with other groups as assessed by reverse transcription-polymerase chain reaction (RT-PCR). In summary, our results showed that Gamat Hydrogel promoted burn wound repair via a complex mechanism involving stimulation of tissue regeneration and regulation of pro-inflammatory cytokines. The resultant wound healing effects were attributed to the synergistic effect of the hydrogel matrix and incorporated sea cucumber.
\end{abstract}

Keyword: Burn; Cytokines; Hydrogel; Wound healing; Wound dressing 